\title{
Editorial
}

\section{The California Perinatal Quality Care Collaborative: A Model for National Perinatal Care}

\section{David K. Stevenson, MD Cecele C. Quaintance, RN}

As my colleague, Professor Fuchs concluded, "managed care is here to stay." "Merger and consolidation have been a consequence of managed care, but any added value to medicine has been variable at best and context- or scale-dependent. Moreover, there may be fundamental folly in the merging of teaching hospitals. ${ }^{2}$ However, there has been at least one useful adjunct of managed care that can be delivered on a large scale without the need for corporate mergers or interference with academic medicine. In particular, the concept of continuous quality improvement (CQI) has contributed to important changes in health care delivery. Although much of the initial effort has been focused on improving administrative and support services, CQI is now used routinely to improve clinical care. Introduction of CQI into health care followed the realization that patient care is a complex process and that real improvement in quality requires understanding and revision of total care based on root cause or system analysis and related to risk-adjusted outcomes.

Thus, the care of high-risk mothers and critically ill babies must also be considered as a complex process. This requires the application of a broad array of evolving technologies by teams of workers, operating within large organizational structures that are faced with competing demands for human and fiscal resources. Perinatal morbidity and mortality are key factors of a health care institution's performance, just as they are indicators of a nation's health status.

The California Perinatal Quality Care Collaborative (CPQCC) is an outgrowth of an initiative proposed by the California Association of Neonatologists (CAN) and supported by the David and Lucile Packard Foundation and the State of California Department of Health Services, Maternal and Child Health Branch. The Collaborative exists to improve the health of pregnant women, infants, and children by collecting high-quality information on perinatal outcomes and resource utilization, which will then allow for performance improvement and bench-marking processes in perinatal care and neonatal intensive care units throughout California.

The preliminary planning group was formed in early 1997 and

Department of Pediatrics, Stanford University School of Medicine, Stanford, CA.

Supported in part by the David and Lucile Packard Foundation, and the State of California Department of Health Services, Maternal and Child Health Branch.

Address correspondence and reprint requests to David K. Stevenson, MD, Department of Pediatrics, Stanford University School of Medicine, Stanford, CA 94305-5119. brings together a public-private alliance of stakeholders, including the Maternal and Child Health Branch, California Children's Services, and the Office of Vital Records (all with the California Department of Health Services); the Office of Statewide Health Planning and Development; the Hospital Council; Regional Perinatal Programs of California; the Health Insurance Plan of California; the American College of Obstetricians and Gynecologists; the California Association of Neonatologists; the Pacific Business Group on Health; the David and Lucile Packard Foundation; and the Vermont Oxford Network. This group, along with others, now serves as the Executive Committee of the Collaborative.

The Collaborative's goal is to improve the quality and outcomes of perinatal health care in California through the following objectives: (1) allow for the timely analysis of perinatal care, outcomes, and resource utilization based upon a uniform statewide data base; (2) provide mechanisms for bench-marking and continuous quality improvement activities; and (3) serve as a model for other states. Further support from the David and Lucile Packard Foundation, the Maternal and Child Health Branch, and California Children's Services has allowed the Collaborative to refine its organizational structure, to establish rules of governance, and to develop data collection and analysis systems and basic performance improvement structures. The Collaborative has broad support from maternal and child health leadership in both public and private sectors throughout the state and is in a prominent position to influence perinatal and neonatal practice and improve the health of women and children in the state of California.

There are currently 37 member hospitals with Neonatal Intensive Care Units (NICUs) admitting nearly $70 \%$ of newborn infants requiring critical care. These member hospitals began submitting data based on a modification of the Vermont 0xford Network neonatal outcomes data system to the Collaborative Data Center. Member hospitals are encouraged to send key clinical leaders to participate in the Collaborative Executive Committee's subcommittees to address development of an infrastructure for performance measurement and improvement, selection of data elements and collection systems, and oversight of risk adjustment and criteria for planned public release of selected performance indicators. The Collaborative is currently focusing on recruiting the 120 hospitals in California with NICUs. After the first few years of development, it is hoped that systems will be in place to add all of the approximately 300 hospitals in California that deliver newborns. It is well-recognized that not all babies with health problems are admitted to an NICU. In order to improve care and outcomes 
for all newborns in California, data for these mothers and newborns will need to be included in the Collaborative data systems and quality improvement systems will need to be expanded.

Of critical importance is the development of an effective quality improvement infrastructure at state, regional, and hospital levels. A permanent subcommittee will review the data and recommend quality improvement objectives, provide models for performance improvement, and assist providers in transforming data into information that can improve care. This committee has recently reviewed a preliminary report on California hospitals to identify both variances in care and outcomes between all California hospitals and other hospitals in the Vermont Oxford Network. Recommendations will be made for a cycle of improvement to test quality improvement structures. Regional programs are assessing CQI capabilities at individual hospitals and identifying potential regional experts. The CPQCC staff has begun work with new members to assess data collection systems and quality of current data submission. The initial focus is on new Collaborative members in the five Regions with agreements to evaluate technical assistance needs and adequacy of the Regional infrastructure to support utilization of information. A CPQCC website is under construction and has basic information about the Collaborative as well as membership documents. As tools for data quality management and quality improvement are developed, they will be added. In addition, reference documents such as care paths, best practices, and other tools, for performance improvement will be posted as they are acquired and/or developed. Other uses of technology, such as a system of electronic dialogue among members, are under consideration with the input of the membership.

The Collaborative Data System is currently based on the Vermont 0xford Network neonatal data system, a voluntary network of hospitals submitting data on very low birth weight babies. The Vermont 0xford Network was selected because of its unique history of data collection, analysis, and quality improvement efforts utilizing simple forms and uniform definitions. In May, California hospitals began data submission on selected larger babies known to be at high risk for serious illness or death. The Collaborative Data Center is working with experts in California and the Vermont Oxford Network to link existing California perinatal data systems to enhance existing data. California is fortunate to have an automated birth and death file containing potentially important information to improve perinatal outcomes. In addition, all California hospitals participate in data collection for the Office of Statewide Health Planning, which includes maternal and newborn discharge, rehospitalization, and costs of care information. When fully implemented, the Collaborative Data Center will provide quarterly hospital and data quality reports with risk-adjusted outcomes comparing similar hospitals to state norms. All reports with comparative data are blinded except for a hospital's own data.

The development of this Collaborative is dependent upon care providers who are actively engaged in the development of both quality improvement, data collection, and analysis systems. The integrity and quality of the data is of utmost importance as well as a belief that risk adjustment is sufficient to account for California's diverse populations and health care models. Competition among providers in California is substantial, and confidentiality during development of data and performance improvement systems is critical in maintaining the focus on improving care and outcomes.

Nonetheless, some kind of "report card" for public dissemination is an important deliverable objective of the project. The format of such reporting and the time of release relative to the CQI process is a sensitive issue. Report cards are a demand of industry and the public in general. However, it is imperative to have physicians involved in picking the indicators, interpreting the risk-adjusted outcomes, designing the format of reporting, and timing the release of information. In the end, the improvement of care for mothers and babies is the Collaborative goal, with the highest quality of care appropriate for the problem, delivered most efficiently at the lowest price. This consequence of managed care can be endorsed and nurtured. Its cause, however, remains ambiguous, with links to the manufacturing industry as well as to the Hippocratic oath.

\section{References}

1. Fuchs VR. Commentary: Managed care and merger mania. JAMA 1987;277: $320-1$.

2. Andreopoulos S. The folly of teaching-hospital mergers. N Engl J Med 1997;336: 61-4. 